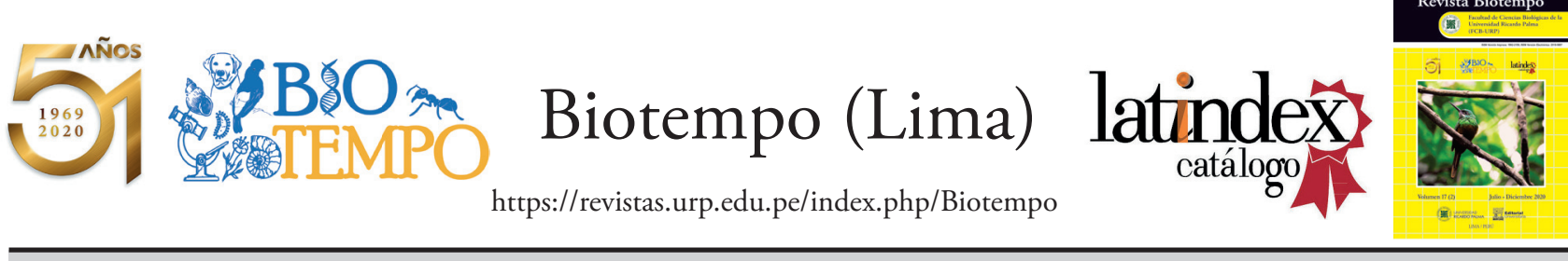

ORIGINAL ARTICLE / ARTÍCULO ORIGINAL

POTENTIAL FOOD ITEMS FOR THE ANDEAN FLAMINGO

(PHOENICOPARRUS ANDINUS, (PHILIPPI, 1854)) IN TWO TYPES OF AQUATIC HABITATS FROM THE PARINACOCHAS LAKE, AYACUCHO, PERU

\title{
ÍTEMS ALIMENTICIOS POTENCIALES PARA LA PARINA GRANDE (PHOENICOPARRUS ANDINUS, (PHILIPPI, 1854)) EN DOS TIPOS DE HÁBITATS ACUÁTICOS DE LA LAGUNA DE PARINACOCHAS, AYACUCHO, PERÚ
}

\section{Enver Ortiz ${ }^{1,2,}$; Miriam Gamboa ${ }^{3}$ Mónica Salas ${ }^{4} \&$ José Vera $^{5}$}

\author{
División de Ornitología, Centro de Ornitología y Biodiversidad - CORBIDI \\ Grupo de Conservación Flamencos Altoandinos - GCFA \\ División de Limnología, Centro de Ornitología y Biodiversidad - CORBIDI \\ Consultora Independente. \\ Peruvian Agency of Cooperation - PERAC. \\ * Corresponding author: eortiz@corbidi.org
}

\section{ABSTRACT}

The presence of a certain species in a place obeys several factors; one of them is the available food resources. In this work, we report the main and potential food items for the Andean Flamingo Phoenicoparrus andinus (Philippi, 1854) would have access to at the end of the winter season in the Parinacochas Lake, Ayacucho in Peru. 21 types of microalgae were found in water associated with shallow puddles and 15 in free surface water: 17 were diatoms (Bacillariophyta), two dinoflagellates (Dinophyceae), and two cyanobacteria (Cyanophyceae) with potential contributions to the diet of $80.96 \%, 9.52 \%$, and $9.52 \%$, respectively. Surirella sp. was present in all the water samples, and it was also the most abundant, followed by the oscillatoriales that were found in $90 \%$ of the samples. Between the two types of habitats, there were more than 12 shared species were registered ( $60 \%$ of similarity) and concerning the distribution of the microalgae in both habitats, the conformation of two undifferentiated groups was observed, this was due to several factors such as the salinity to which they were exposed and the proximity to hot springs, which could influence the distribution and therefore availability and accessibility of microalgae as potential food items for the Andean Flamingo in the northernmost site of its distribution in South America.

Keywords: Flamingos - habitat use - Parinacochas - Potential food - The Andes - trophic ecology - winter season 


\section{RESUMEN}

La presencia de una determinada especie en un lugar obedece a varios factores, uno de ellos son los recursos alimenticios disponibles. En este trabajo registramos los principales y potenciales ítems alimenticios a los que tendría acceso la Parina Grande Phoenicoparrus andinus (Philippi, 1854) a finales de invierno en la laguna de Parinacochas, al sur de Ayacucho en Perú. Se hallaron 21 tipos de microalgas en agua asociada a charcos de poca profundidad y 15 en agua superficial libre: 17 fueron diatomeas (Bacillariophyta), dos dinoflagelados (Dinophyceae) y dos cianobacterias (Cyanophyceae) con potenciales contribuciones a la dieta del 80,96\%, 9,52\% y 9,52\% respectivamente. El género Surirella sp., estuvo presente en todas la muestras de agua además fue el más abundante, seguido de los oscillatoriales que estuvieron en el $90 \%$ de las muestras. Entre los dos tipos de hábitats se registraron más de 12 especies compartidas (60\% de similaridad); y con respecto a la distribución de las microalgas en ambos hábitats, se observó la conformación de dos grupos no diferenciados, esto se debería a varios factores como la salinidad a la que se encentraban expuestas y la proximidad a fuentes termales, las que podrían influir en la distribución y por lo tanto disponibilidad y accesibilidad de las microalgas como potenciales ítems alimenticios para la Parina Grande en el sitio más septentrional de su distribución en Sudamérica.

Palabras clave: Alimento potencial - ecología trófica - flamencos - invierno - los Andes - Parinacochas - uso del hábitat

\section{INTRODUCCIÓN}

La Parina Grande o Parihuana Andina (Phoenicoparrus andinus (Philippi, 1854)) ocurre comúnmente en los lagos salinos tanto de los altos Andes como de áreas pre andinas de Argentina, Bolivia, Chile y Perú (Goodall et al., 1951; Hurlbert \& Keith, 1979; Fjeldså \& Krabbe, 1990; Parada, 1990; Araya \& Millie, 2005; Jaramillo, 2005; Tobar et al., 2014). En Perú la especie es considera como visitante no reproductivo en el suroeste del Perú (Schulenberg, 2010). Siendo registrada en tres sitios altoandinos habituales: Laguna Loriscota (Puno), Salinas (Arequipa) y Parinacochas (Ayacucho) (Koepcke \& Koepcke, 1952; Hurlbert \& Keith, 1979; Valqui et al., 2000; Ricalde, 2003; Angulo, 2009; eBird, 2020).

Durante el invierno las Parinas Grandes se desplazan hacia lagunas y humedales de tierras bajas, en búsqueda de mejores condiciones de hábitat; en Argentina, laguna Melincué (34² $42^{\prime} \mathrm{S} 61^{\circ} 29^{\prime} \mathrm{O} ; 84 \mathrm{msnm}$ ) (Romano et al., 2002), en humedales costeros como el de la desembocadura del río Mataquito, región del Maule, centro de Chile ( $35^{\circ} 18^{\prime} \mathrm{S} 72^{\circ} 24^{\prime} \mathrm{O}$; 0 msnm) (González et al., 2012). Y en Perú, también en la costa sur del país; uno de los primeros registros documentados corresponde a diez individuos entre adultos y juveniles en los humedales de Ite en Tacna ( $17^{\circ} 53^{\prime} \mathrm{S} 70^{\circ} 59^{\prime} \mathrm{O}$; 0 msnm) en enero de 2009 por Vizcarra et al., (2009); además del registro de Høgsås et al. (2010) en el Santuario Nacional Lagunas de Mejía en Arequipa
(17 $8^{\circ} \mathrm{S} 71^{\circ} 51^{\prime} \mathrm{O}$; $8 \mathrm{msnm}$ ), siendo todos estos registros asincrónicos y oportunistas.

En todos estos ambientes, sobre todo los altoandinos, es común encontrar diversidad de microrganismos acuáticos como copépodos, cladóceros, coríxidos, anfípodos; y algas como bacilariofitas en altas densidades (Hurlbert \& Keith, 1979), inicialmente la dieta de la Parina Grande fue descrita mediante el análisis de heces, estando constituida en gran proporción por las frústulas de diatomeas ( $>80 \mu \mathrm{m}$ de longitud) como lo describió Hurlbert en 1982; La Parina Grande ha sido considerada omnívora, pudiendo consumir diatomeas de gran tamańo de los géneros Navicula sp., Surirella sp. y Amphora sp.; Y también de microinvertebrados entre los que resaltan larvas de insectos, nemátodos y copépodos de menor tamaño (Gallardo \& Rodríguez, 1992; Rodríguez, 2005).

Tiempo después, en el 2012 la dieta fue redescrita específicamente por el consumo de Surirella sella (Schmidt, 1925), Denticula thermalis (Kützing, 1844), Pinnularia sp. Mastogloia sp. y Haloroundia speciosa (Hustedt, 1927) (Tobar et al., 2014), un par de años después se reportó el consumo de otras especies del género Surirella sp., las que varían entre $60-250 \mu \mathrm{m}$ de longitud, además de pequeños nemátodos de entre 100 - $300 \mu \mathrm{m}$, pequeños protozoarios y crustáceos de entre $40-130 \mu \mathrm{m}$ inmersas en aguas con salinidad variable entre 5 y $100 \mathrm{~g} / \mathrm{L}$. (S. Hurlbert, comunicación personal, 7 de octubre de 2014). 
Finalmente un estudio mucho más detallado en 2018, concluyó y corroboró, al igual que los trabajos anteriores, que la especie se alimenta principalmente de microalgas como las diatomeas Surirella striatula (Turpin, 1828) y Surirella ovalis (Brébisson, 1838), las que fueron halladas en sus heces en gran proporción, además el estudio concluyó existe una fuerte selección positiva alimenticia hacia el grupo de las diatomeas y una negativa hacia los microinvertebrados (Polla et al., 2018).

El objetivo de este trabajo es proporcionar información cualitativa sobre cuáles son las microalgas, como principales ítems alimenticios, que estaría consumiendo la Parina Grande en dos tipos de hábitats acuáticos en la laguna de Parinacochas, Ayacucho, Perú durante su residencia en época invernal.

\section{MATERIALES Y MÉTODOS}

\section{Área de estudio}

A principios de agosto de 2015 (último mes del invierno austral), visitamos la laguna de Parinacochas $\left(15^{\circ} 17^{\prime} \mathrm{S}, 73^{\circ} 41^{\prime} \mathrm{O}, 3278 \mathrm{~m}\right)$ al sur de Ayacucho (Fig. $1)$, esta laguna cuenta con una extensión que varía entre los 64 y los $67 \mathrm{~km}^{2}$ durante temporada de lluvias (Koepcke \& Koepcke, 1952; Hurlbert et al., 1986) y pudiendo retraerse hasta $58 \mathrm{~km}^{2}$ durante la época seca; es de aguas poco profundas, hasta 1,5 m (GRRNGMA, 2014) y con una salinidad de 5,6 g / L, (Hurlbert et al., 1986), dicha concentración de sal se debe como en la mayoría de lagos salinos altoandinos a la evaporación del agua y a la concentración sucesiva de sales, típicos fenómenos presentes en cuencas endorreicas. Dicha área se caracteriza por ser de clima frio durante la temporada de invierno y en verano con abundantes lluvias, la temperatura mínima multianual varía entre los 11 y los $16{ }^{\circ} \mathrm{C}$, y las precipitaciones varían en periodo lluvioso (verano) entre los 250 y los $519 \mathrm{~mm}$ (GRRNGMA 2014).

\section{Muestreo y Procesamiento}

Se tomaron muestras, siguiendo indicaciones generales de Tobar et al. (2014), tanto de agua asociada a charcos, con una profundidad de hasta $10 \mathrm{~cm}$ (1A) y agua superficial libre, de más de $50 \mathrm{~cm}$ de profundidad (1B); cinco muestras por sitio en un solo transecto tomados paralelamente a la orilla, con una separación entre ellos de $30 \mathrm{~m}$, cada punto rojo representa una muestra (Fig. 1), este arreglo en la metodología de muestreo se debió a que previamente se verificó la presencia y uso del hábitat durante la alimentación por parte de las Parinas Grandes el día anterior al muestreo y con ellos se buscó que el muestreo tuviera una adecuada representatividad, estos sitios en ambos casos fueron cercanos a la orilla $(80-100 \mathrm{~m}$ de distancia aproximadamente).

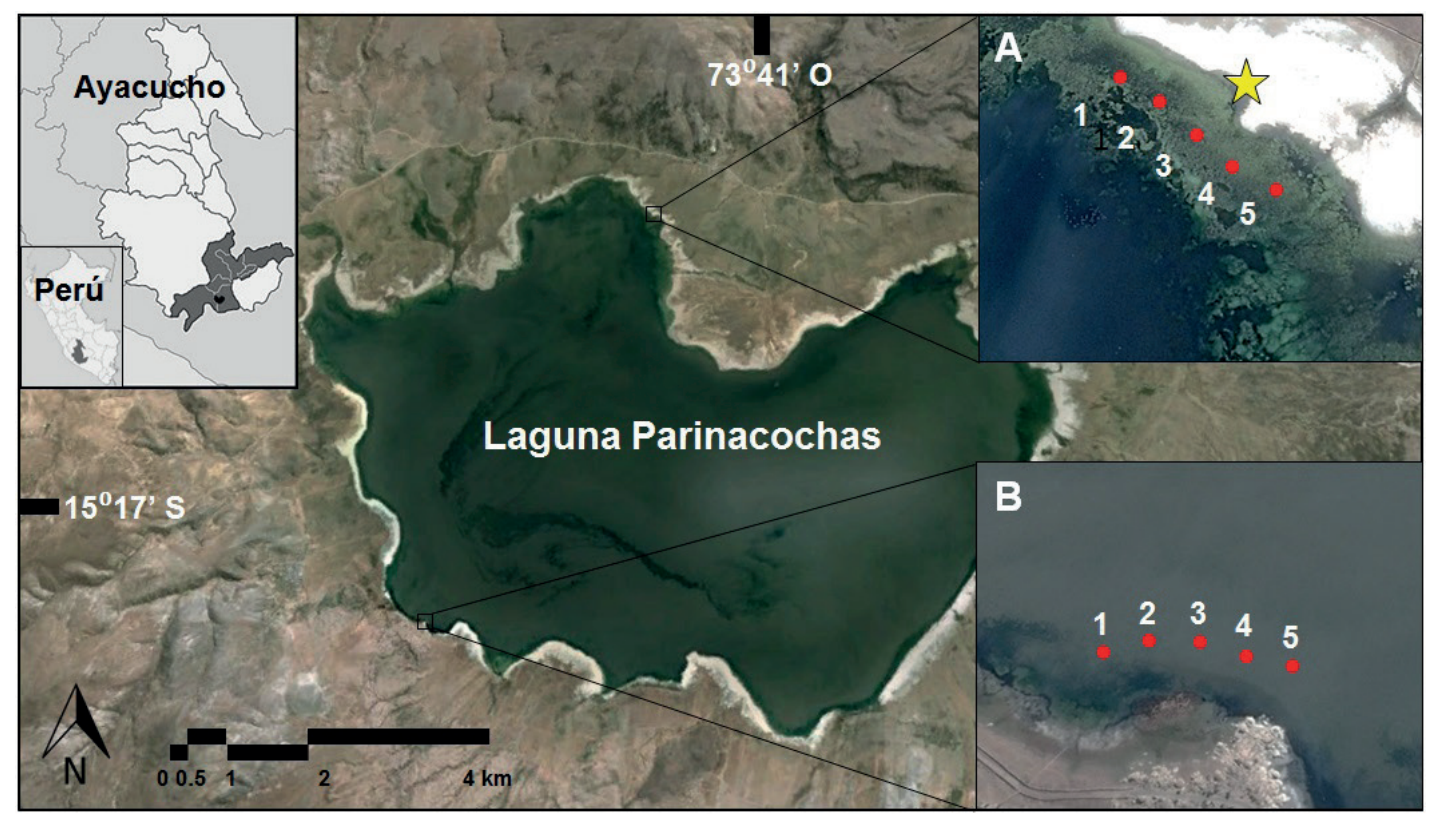

Figura 1. Área de estudio, laguna de Parinacochas al sur de Ayacucho, localizada a 3278 msnm, además se muestran las zonas de muestreo 1A: agua asociada a charcos de poca profundidad y 1B: agua superficial libre. La estrella amarilla representa la fuente termal adyacente a la zona de muestreo. 
Las muestras fueron colectadas entre las 7:00 y 7:30 am en $1 \mathrm{~A}$ y entre las 2:00 y 2:30 pm en $1 \mathrm{~B}$. Por cada muestra se tomaron $45 \mathrm{~mL}$ de agua asociada a charcos y agua superficial libre directamente con una jeringa y preservándolas en formol al $4 \%$. Esta toma directa de muestras se realizó debido a que se carecía de redes adecuadas para realizar el filtrado y tamizado. Sin embargo esta fueron estandarizadas en el análisis microscópico de acuerdo con (Tobar et al., 2014).

El análisis taxonómico en laboratorio fue hecho usando un microscopio óptico con 200x de aumento, además se hizo uso de fotografías digitales para las identificaciones integrando cámara digital al ocular del microscopio, manteniendo las dimensiones de las identificaciones, las cuales fueron medidas usando el ocular del micrómetro graduado. En una laminilla cubreobjetos de 32x25 mm. Se precipitó una alícuota de $0,05 \mathrm{~mL}$ de cada muestra. Posteriormente se hizo una lectura en barrido horizontal de tres transectos (Villafañe \& Reid, 1995; Tobar et al., 2014) para evitar el efecto de frontera (Fabricius et al., 2005). Para efectos específicos solo se identificaron microalgas en el orden de 40 - $300 \mu \mathrm{m}$ debido que las Parinas Grandes filtran y se alimentan de microalgas en dichos rangos de longitud (Tobar et al., 2014; Polla et al., 2018).

Para la identificación de los taxones se usó guías pertinentes (Díaz \& Maidana, 2005; Tobar et al., 2014). El análisis estadístico consistió en la aplicación del índice de Similaridad de Sorensen (Tobar et al., 2014) entre las muestras de agua de cada una de las estaciones de los dos grupos (agua asociada a charcos de poca profundidad y agua superficial libre). Después se usó el análisis de coordenadas principales (Gower, 2014), como método para representar gráficamente la distribución de las microalgas encontradas en cada hábitat y al mismo tiempo se calculó la afinidad entre ambos tipos de hábitats de donde se tomaron las muestras. Ambos análisis se ejecutaron en la versión 2.16 PAST (Paleontological statistics software package for education and data analysis) (Hammer et al., 2001).

\section{Aspectos éticos}

La información obtenida se utilizó solo para los fines de la presente investigación; no implicó ningún tipo de afectación personal, social u ecológica antes, durante y después de la investigación.

\section{RESULTADOS}

A finales del invierno del 2015, en muestras de agua provenientes dos tipos de hábitats en la laguna de Parinacochas, se identificaron un total de 21 potenciales ítems alimenticios en agua de charcos de poca profundidad y 15 en agua superficial libre para la Parina Grande (P. andinus) (Tabla 1). Las que se distribuyeron en tres taxones: las diatomeas (Bacillariophyta) con el 80,96\%, dinoflagelados (Dinophyceae) y cianobacterias (Cyanophyceae) con 9,52\% cada uno (Fig. 2). Dentro de las diatomeas, el orden Centrales congregó a la mayoría de ellas, con un total de 11 especies; dentro de este orden, dos géneros estuvieron presentes en las mayorías de las muestras: Nitzschia sp. en siete y Navicula sp. en seis de un total de diez muestras que se tomaron. Mientras que el orden Pennales estuvieron representados por seis especies de diatomeas, el género Surirella sp.; además estuvo presente en todas las muestras (Tabla 1). En el caso de los dinoflagelados Peridinium sp. estuvo presente en cuatro de las cinco muestras de aguas asociadas a charcos, mientras que en el caso de las cianobacterias el orden oscillatoriales estuvo presente en nueve de las diez muestras, en cuatro de agua asociadas a charcos y en todas las de superficie libre.

En tanto que en las muestras provenientes de agua asociada a charcos tuvieron mayor porcentaje de presencia, de por lo menos algún tipo de microalgas, en las cinco muestras tomadas lo que representó el $63,63 \%$ del total a comparación de las muestras de agua superficial libre que solo obtuvieron un $36,37 \%$. Cabe mencionar que la muestra $3 \mathrm{~A}$, fue la que mayor diversidad presentó, 14 de las 21 especies de microalgas registradas, entre todas las demás muestras tomadas en ambos tipos de hábitats.

En lo referido a la similaridad de entre las muestras, cuatro estaciones de agua asociada a charcos de poca profundidad, compartieron más del $50 \%$ de microrganismos (Fig. 3); en otras palabras tenían en común entre 12 a 13 de las 21 especies de microalgas registradas, lo mismo sucedió en las muestras de agua superficial libre, pero en este caso sólo fueron tres muestras. Con respecto a la distribución de las microalgas (Fig. 4), se encontró que si bien no forman grupos definidos, ya que se observa una superposición, se evidenció que existe un nivel de similaridad entre ambos grupos. 


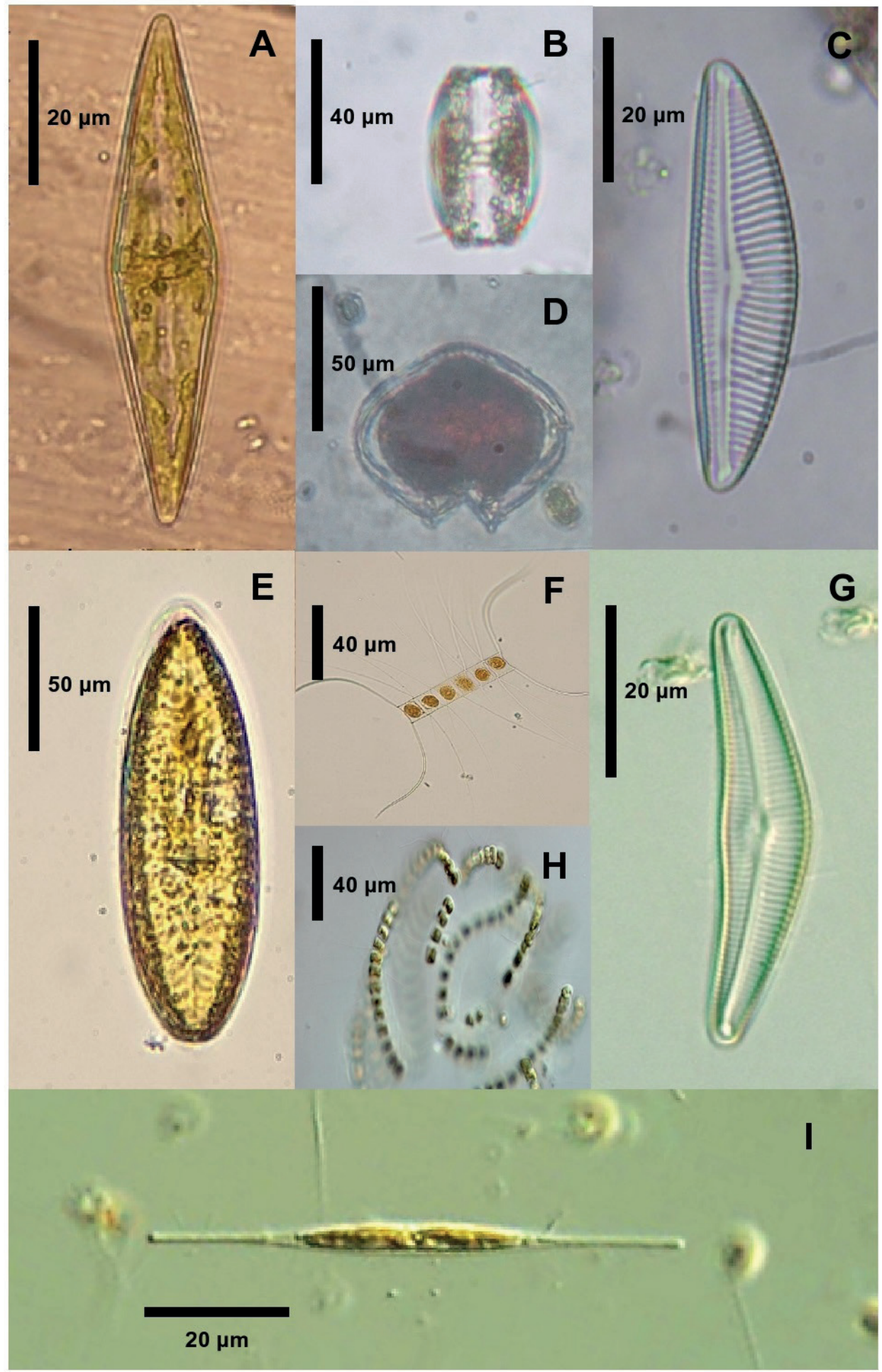

Figura 2. Principales microalgas encontradas tanto en agua asociadas a charcos de poca profundidad como en agua superficial libre en la Laguna de Parinacochas al sur de Ayacucho, en agosto de 2015. A: Pleurosigma sp., B: Thalassiosira sp., C: Encyonema sp., D: Peridinium sp. (Dinophyceae), E: Surirella sp., el más abundante entre todas los microalgas halladas. F: Chaetoceros affinis, G: Cymbella sp., H: Chaetoceros sp. e I: Nitzschia sp., la mayoría de ellos son diatomeas excepto se mencione otra taxa. 
Tabla 1. Lista de todas las microalgas encontradas y frecuencias en dos tipos de hábitats acuáticos de la Laguna de Parinacochas al sur de Ayacucho.

\begin{tabular}{|c|c|c|}
\hline \multirow[b]{2}{*}{ Taxa / Orden / Especie } & \multicolumn{2}{|c|}{ Frecuencia de ocurrencia (\%) } \\
\hline & $\begin{array}{c}\text { Asociada a } \\
\text { charcos }\end{array}$ & $\begin{array}{l}\text { Superficial } \\
\text { libre }\end{array}$ \\
\hline \multicolumn{3}{|l|}{ Bacillariophyta (Diatomeas) } \\
\hline \multicolumn{3}{|l|}{ Centrales } \\
\hline Chaetoceros affinis (Lauder, 1864) & 60 & 20 \\
\hline Chaetoceros decipiens (Cleve, 1873) & 10 & - \\
\hline Chaetoceros didymus (Ehrenberg, 1845) & 40 & 20 \\
\hline Chaetoceros socialis (Lauder, 1864) & 60 & - \\
\hline Cymbella sp. (Agardh, 1830) & 40 & 20 \\
\hline Detonula pumila (Peragallo, 1888) & 20 & 20 \\
\hline Encyonema sp. (Kützing, 1833) & 80 & - \\
\hline Hemiaulus sinensis (Greville, 1865) & 20 & 20 \\
\hline Lithodesmium undulatum (Ehrenberg, 1839) & 40 & 50 \\
\hline Navicula sp. (Bory de Saint-Vincent, 1822) & 40 & 60 \\
\hline Nitzschia sp. (Hassall, 1845) & 80 & 60 \\
\hline \multicolumn{3}{|l|}{ Pennales } \\
\hline Pleurosigma sp. (Smith, 1852) & 20 & - \\
\hline Pseudonitzschia Grupo seriata (Peragallo, 1899) & 40 & 40 \\
\hline Rhopalodia sp. (Müiller, 1895) & 40 & - \\
\hline Surirella sp. (Turpin, 1828) & 100 & 100 \\
\hline Skeletonema costatum (Greville, 1866) & 20 & - \\
\hline Thalassiosira sp. (Cleve, 1873) & 40 & 20 \\
\hline \multicolumn{3}{|l|}{ Dinophyceae Dinoflagelados ) } \\
\hline \multicolumn{3}{|l|}{ Prorocentrales } \\
\hline Prorocentrum sp. (Ehrenberg, 1834) & 20 & 20 \\
\hline \multicolumn{3}{|l|}{ Peridiniales } \\
\hline Peridinium sp.(Ehrenberg, 1830) & 80 & 20 \\
\hline \multicolumn{3}{|l|}{ Cyanophyceae (Cianobacterias) } \\
\hline Chroococcales & 40 & 20 \\
\hline Oscillatoriales & 80 & 100 \\
\hline Número total de taxones & 21 & 15 \\
\hline
\end{tabular}




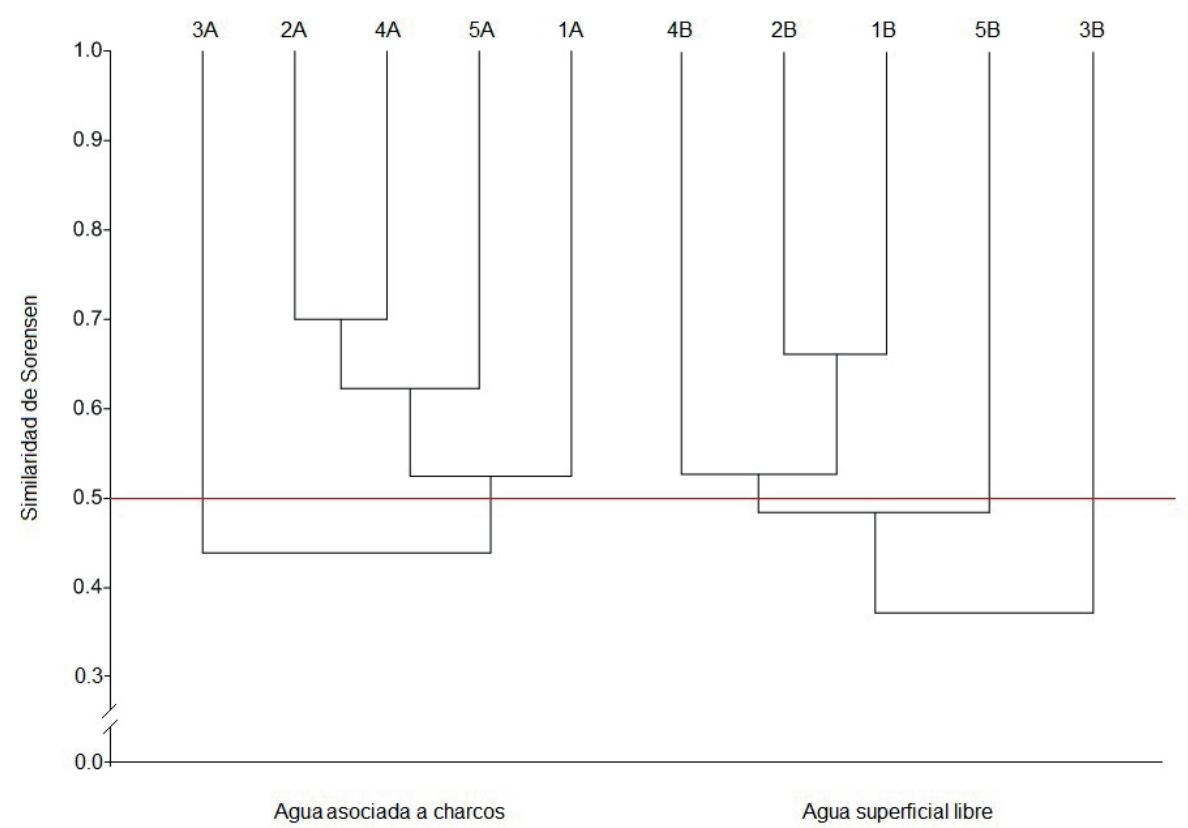

Figura 3. Dendrogramas para el índice de similaridad de Sorensen para ambos hábitats: agua asociada a charcos de poca profundidad y agua superficial libre, de cada uno de ellos se tomaron cinco muestras.



PC 1 (37.13\% de la variación total)

Figura 4. Análisis de coordenadas principales para la ordenación por similaridad de los microrganismos provenientes dos tipos de hábitats: agua asociada a charcos de poca profundidad y agua superficial libre. (Círculos rojos: Diatomeas, verdes: Cianobacterias y azules: Dinoflagelados) 


\section{DISCUSIÓN}

Este trabajo, al igual que investigaciones previas realizadas en zonas altoandinas, muestra que la presencia de diatomeas en estos ambientes son los ítems alimenticios por excelencia para la Parina Grande esto ha sido reportado desde Hurlbert \& Keith (1979) hasta Polla et al. (2018). La diversidad que mostramos aquí para este grupo de microalgas es casi igual a la reportada por López (1991) en el salar de Carcote $\left(21^{\circ} 15^{\prime}\right.$ S, 68²0' $\mathrm{O}, 3690 \mathrm{~m}$.) norte de Chile, quien halló 16 especies, pero es inferior a la reportadas por Tobar et al. (2014) en el salar de Punta Negra $\left(24^{\circ} 35^{\prime}\right.$ S, 68 $58^{\prime}$ O, 3000 m.) en la región de Antofagasta con 39 especies y Polla et al. (2018) en el sistema de humedales de Pampa de las Lagunas $\left(33,70^{\circ}-34,30^{\circ} \mathrm{S}, 61,42^{\circ}-62,53^{\circ} \mathrm{O}, 80\right.$ $130 \mathrm{msnm}$ ) en la región central este de Argentina con 34 especies de diatomeas; determinándose en estos dos últimos casos las contribuciones de la dieta fueron de $100 \%$ y $97 \%$, respectivamente; nuestro trabajo muestra que el grupo de las diatomeas contribuiría solo al $70 \%$ de la dieta; sin embargo esto no es concluyente debido a que el restante de los ítems siguen siendo potenciales y desconocemos si las Parinas Grandes las discriminaran o no al momento de alimentarse.

Nosotros creemos, al igual que Tobar et al. (2014), que la presencia de la Parina Grande en la laguna de Parinacochas se deba principalmente a la presencia de las diatomeas; aquí reportamos al género Surirella sp., como uno de los ítems potenciales más abundantes presentes en todas las muestras colectadas, esto concuerda con los hallazgos de Hurlbert \& Chang (1983) quienes registraron, en laguna Puripica Chico al sur de Bolivia (22³1’ S, 67³0’ O, 4393 $\mathrm{m}$.$) , que el consumo predominante era Surirella wetzeli$ (Hustedt, 1925), mientras que López (1991) reportó que Parinas Grandes en el salar de Carcote consumían frecuentemente $S$. sella. Tobar et al. (2014) registraron que la misma especie de diatomea era consumida abundantemente por Parinas Grandes en el salar de Punta Negra, corroborando lo hallado previamente por Gallardo \& Rodríguez (1992) en el salar de Surire (1850'14" S, 6902'46" O, $4250 \mathrm{msnm}$ ), ambos sitios al norte de Chile. Finalmente, Polla et al. (2018) encontraron que $S$. striatula y $S$. ovalis además de otras diatomeas estuvieron presentes en gran volumen en las heces, lo que indica que este grupo de microalgas compone principalmente la dieta de la Parina Grande en Pampa de las Lagunas en la región central este de Argentina.

Se ha reportado la presencia e incluso abundancia de diatomeas en fuentes termales (Smith et al., 2013; Leira et al., 2017). Aquí reportamos también la presencia y abundancia de cuatro géneros: Encyonema sp., Navicula sp., Nitzschia sp. y Rhopalodia sp., en muestras agua asociadas a charcos, las cuales estuvieron cerca de una fuente termal (2A, 3A y 4A), ver Fig. 1. Asimismo, en el caso de las cianobacterias, fueron reportadas en fuentes termales, si bien la temperatura en promedio fue de $34 \mathrm{a}$ $38^{\circ} \mathrm{C}$, esto puede cambiar el dominio de la flora algal de diatomeas hacia las cianobacterias (Patrick et al., 1969), aquí reportamos también la presencia y abundancia de los oscillatoriales (Cianobacterias) sobre todo en la muestra $3 \mathrm{~A}$, la más cerca a la fuente termal, sin embargo esto no afectó al abundancia de las diatomeas, solo contribuyeron en un $7,5 \%$ a los potenciales ítems alimenticios, resaltándose que fue en esta muestra donde el género Surirella sp., fue más abundante que en las otras muestras, posiblemente esto dependa de otros factores como los rangos óptimos de tolerancia a la salinidad (Potapova, 2011); así como otros parámetros fisicoquímicos de la columna de agua, que inducen cambios en la composición de la comunidad de microorganismos (Núnez-Salazar et al., 2020), siendo estos factores no tomados en cuenta en este trabajo.

Con respecto a la presencia de las microalgas entre los dos tipos de hábitats, si bien existió una similaridad entre el 50 y $60 \%$ de especies, esto se debería al efecto de la presencia compartida de muchas especies de microalgas entre los hábitats (Mesnage et al., 2006). La conformación de los grupos pudiera verse afectada por la alta abundancia de Surirella sp., la que estuvo presente en todas las muestras y la de los oscillatoriales en nueve de ellas. Otro caso destacable es la ocurrencia de Encyonema sp. y Chaetoceros sp., solamente en las muestras de agua asociada a charcos, otro hecho que podría afectar conformación de los grupos es la baja o nula ocurrencia de microalgas en las muestras de agua superficial libre (Tabla 1).

Finalmente las muestras de agua superficial tuvieron una abundancia relativamente baja; asimismo en alguna de ellas, algunas especies de microalgas estuvieron ausentes (Tabla 1), en consecuencia sugerimos que esto puede deberse a la altura de la columna de agua de donde se colectó la muestra, en algunos casos fue de casi $50 \mathrm{~cm}$, esto podría estar generando un efecto de 'dilución' de los microorganismos (Mesnage et al., 2006), haciendo del hábitat poco preferible para las Parinas Grandes, ya que de esta manera les tomaría más tiempo en filtrar las pocas microalgas presentes, provocando que las Parinas Grandes prefieran alimentarse en lagunas y salares de poca profundidad $(<40 \mathrm{~cm})$, al igual que las otras especies de flamencos, como ha sido reportado por Canevari (1983), donde se espera que la concentración de microalgas sea mucho mayor (Polla et al., 2018). 


\section{AGRADECIMIENTOS}

Los autores desean expresar sus agradecimientos a las personas que contribuyeron a este trabajo, ya sea desde compartir bibliografía especializada y a las personas que apoyaron en las coordinaciones logísticas para poder salir a campo y así poder muestrear.

\section{REFERENCIAS BIBLIOGRAFICAS}

Angulo, F. 2009. Important bird areas Americas - Priority sites for biodiversity conservation, Perú. En BirdLife Conservation Series, 16. Devenish, C.; Díaz-Fernández, D.F; Clay, R.P.; Davidson, I.J. \& Yépez-Zabala, Í. (eds.). Quito, Ecuador, BirdLife International. pp. 307-316.

Araya, B.M. \& Millie, G.H. 2005. Guía de campo de las aves de Chile. $10^{\text {th }}$ ed. Editorial Universitaria, Santiago, Chile.

Canevari, P. 1983. El Flamenco Común. Centro Editor de América Latina, Buenos Aires, Argentina.

Díaz, C. \& Maidana, N.I. 2005. Diatomeas de los Salares Atacama y Punta Negra. II Región, Chile. Centro de Ecología Aplicada Ltda., Santiago, Chile.

eBird. 2020. eBird: An online database of bird distribution and abundance [web application]. eBird, Ithaca, New York. Available: https://www.ebird.org (Accedido 14/7/2020)

Fabricius, A.L.; Luque, M.E., \& Bocolini, M. 2005. Diatomeas planctónicas de cursos de agua. Cuenca del Río Piedra Blanca (Córdoba, Argentina). Boletín de la Sociedad Argentina de Botánica, 40:183-198

Fjeldså, J. \& Krabbe, N. 1990. Birds of the high Andes. Copenhagen: Zoological Museum Univerrsity of Copenhagen \& Svendborg: Apollo Books. Svendborg, Denmark, 880 pp.

Gallardo, O.P. \& Rodríguez, E. 1992. Hábitos alimentarios y etología de flamencos en el Salar de Surire. Tesis de grado. Universidad Arturo Prat. 89 pp.

González, A.; Vukasovic, M.; López, V. \& Estades, C. 2012. Variación temporal de la abundancia y diversidad de aves del humedal del río Mataquito, Región del Maule, Chile. Hornero 27: 167-176.

Goodall, J.D.; Johnson, A.W. \& Philippi, R.A. 1951. Las aves de Chile, su conocimiento y sus costumbres.
Tomo II. Platt Establecimientos Gráficos. Buenos Aires, Argentina.

Gower, J.C. 2014. Principal Coordinates Analysis. Wiley StatsRef: Statistics Reference Online.

Gerencia de Recursos Naturales y Gestión Del Medio Ambiente (GRRNGMA). 2014. Expediente técnico para la propuesta de establecimiento de la laguna Parinacochas - Ayacucho como Área de Conservación Regional. Gobierno Regional de Ayacucho. Ayacucho. 189 pp.

Hammer, Ø.; Harper, D. \& Ryan, P. 2001. PAST: Paleontological statistics software package for education and data analysis. Electronic Paleontology, 4: 1-9.

Høgsås, T.; Vizcarra, J.; Hidalgo N. \& Málaga E. 2010. Primeros registros documentados de Phoenicoparrus andinus en la costa sur de Perú. Cotinga, 32:155-157.

Hurlbert, S.H. \& Keith, J.O. 1979. Distribution and spatial patterning of flamingos in the Andean Altiplano. Auk 96: 328-342.

Hurlbert, S.H. 1982. Limnological studies of flamingo diets and distributions. National Geographic Society Research Report. 14:351-356.

Hurlbert, S.H. \& Chang, C.C.Y. 1983. Ornitholimnology: Effects of grazing by the Andean flamingo (Phoenicoparrus andinus). Proceedings of the National Academy of Science USA 80: 47664769 .

Hurlbert, S.H.; Loayza, W. \& Moreno, T. 1986. Fishflamingo-plankton in the Peruvian Andes. Limnology and Oceanography, 31:457-468.

Jaramillo, A. 2005. Aves de Chile. Lynx Ed. Barcelona, Spain.

Koepcke, H. \& Koepcke. M. 1952. El Lago Parinacochas región que debe convertirse en "Parque Nacional". Pesca y Caza, 5:23-30.

Leira, M.; Meijide-Failde, R. \& Torres, E. 2017. Diatom communities in thermo-mineral springs of Galicia (NW Spain), Diatom Research, 32: 2942.

López, M. 1991. Alimentación de flamencos altiplánicos con énfasis en Phoenicoparrus andinus (Phillippi) en el salar de Carcote, Chile. En: I Taller Interna- 
cional de especialistas en flamencos sudamericanos. Corporación Nacional Forestal de Chile \& Sociedad Zoológica de Nueva York, pp. 84-89.

Mesnage, V.; Ogier, S.; Bally, G.; Disnar, J. R.; Lottier, N.; Dedieu, K.; Rabouille, C. \& Copard, Y. 2006. Nutrient dynamics at the sediment-water interface in a Mediterranean lagoon (Thau, France): Influence of biodeposition by shellfish farming activities. Marine Environmental Research 63: 257-277

Núñez-Salazar, R.; Aguirre, C.; Soto, J.; Salinas, P.; Salinas, C.; Prieto, H. \& Paneque, M. 2020. Physicochemical parameters affecting the distribution and diversity of the water column microbial community in the high-altitude Andean lake system of La Brava and La Punta. Microorganisms, 8: 1181.

Patrick, R.; Crum, B. \& Coles, J. 1969. Temperature and manganese as determining factors in the presence of diatom or blue-green algal floras in streams. Proceedings of the National Academy of Sciences of the USA, 64: 472-478.

Parada, M. 1990. Flamencos en el norte de Chile. Distribución, abundancia y fluctuaciones estacionales del número. En: I Taller Internacional de Especialistas en Flamencos Sudamericanos. Parada, M.; Rottmann, J. \& Guerra C. (eds). Corporación Nacional Forestal de Chile \& Sociedad Zoológica de New York, pp. 52-66.

Potapova, M. 2011. Patterns of diatom distribution in relation to salinity In: Seckbach, J. \& Kociolek, J.P. (eds.), The Diatom World, Cellular Origin, Life in Extreme Habitats and Astrobiology, 19: 313-332.

Polla, W.; Di Pasquale, V.; Rasuk, M.; Barberis, I.; Romano, M.; Manzo, R.; Paggi, J.; Farías, M.; Contreras, M. \& Devercelli, M. 2018. Diet and feeding selectivity of the Andean Flamingo Phoenicoparrus andinus and Chilean Flamingo Phoenicopterus chilensis in lowland wintering areas. Wildfowl, 68: 3-29.

Ricalde, D.G. 2003. Conservación de flamencos en el Altiplano Peruano. Lyonia, 4: 87-93.

Rodríguez, E. 2005. Conservación de Flamencos Altoandinos en el Norte de Chile: Estado actual y plan de conservación. Corporación Nacional Forestal de Chile. 91 pp.

Romano, M.; Pagano, F. \& Luppi, M. 2002. Registros de Parina Grande (Phoenicoparrus andinus) en la Laguna Melincué, Santa Fe, Argentina. Nuestras Aves, 43:15-16.

Schulenberg, T.S.; Stotz, D.F.; Lane, D.F.; O’Neill, J.P., \& Parker. T.A. 2010. Birds of Peru. Princeton University Press. Princeton, New Jersey. 664 pp.

Smith, T.E.; Manoylov, K.M. \& Packard, A. 2013. Algal extremophile community persistence from hot springs National Park (Arkansas, U.S.A.). International Journal on Algae, 15: 65-76.

Tobar, C.; Rau, J.R.; Iriarte A.; Villalobos, R.; Lagos, N.; Cursach, J.; Díaz, C.; Fuentes N. \& Gantz, A. 2012. Composition, diversity and size of diatoms consumed by the Andean Flamingo (Phoenicoparrus andinus) in salar de Punta Negra, Antofagasta Region, Northern Chile. Ornitología Neotropical, 23: 243-250.

Tobar. C.; Rau, J.; Fuentes, N.; Gantz, A.; Cursach, J.; Suazo C.; Santibáñez A. \& Perez-Shultheiss, J. 2014. Diet of the Chilean flamingo Phoenicopterus chilensis (Phoenicopteriformes: Phoenicopteridae) in a coastal wetland in Chiloé, Southern Chile. Revista Chilena de Historia Natural, 87: 15.

Valqui, M.; Caziani, S.M.; Rocha, O. \& Rodríguez, E. 2000. Abundance and distribution of the South American altiplano flamingos. The International Journal of Waterbird Biology, 23, Special Publication 1: Conservation Biology of Flamingos: 110-113.

Villafañe, V.E. \& Reid, F.M. 1995. Métodos de Microscopia para la cuantificación del fitoplancton. In: Alveal, K.; Ferrario, M.E.; Oliveira, E.C. \& Sar, E. (eds). Manual de Métodos Ficológicos. Universidad de Concepción, Concepción.

Vizcarra, K.J.; Hidalgo, N. \& Chino, E. 2009. Adiciones a la avifauna de los Humedales de Ite, costa sur de Perú. Revista Peruana de Biología, 16: 221-225.

Received October 6, 2020.

Accepted October, 27, 2020. 